\title{
The Relationship Between Placental Elasticity and
}

\section{Prenatal Serum Screening Markers and Doppler}

\section{Indices}

\author{
Murat Akbaş $^{1^{\star}}$, Faik Mümtaz Koyuncu${ }^{1}$, Hakan Erenel ${ }^{2}$, Burcu Artunc Ulkumen ${ }^{1}$, Tülay Oludağ \\ Mete $^{1}$
}

'Manisa Celal Bayar University Faculty of Medicine, Department of Obstetrics and Gynecology, Manisa, Turkey
${ }^{2}$ Istanbul University, Cerrahpasa Faculty of Medicine, Department of Obstetrics and Gynecology, Istanbul, Turkey

\begin{abstract}
It has been shown that placental elasticity values were higher in pregnant women with preeclampsia and intrauterine growth restriction. The studies have been reported that prenatal serum screening markers and uterine artery Doppler parameters could be useful in the prediction of preeclampsia and intrauterine growth restriction. In this study, we aimed to investigate the relationship between placental elasticity values and these serum markers and Doppler indices in the first and second-trimester.

108 cases between 11-14 gestational weeks were enrolled for this study. Placental elasticity measurements were performed after the first-trimester combined test and Doppler assessment. The same procedures were repeated with the secondtrimester triple test for 71 pregnant women who were followed-up. Correlation analysis of demographic characteristics, biochemical parameters and Doppler findings with placental elasticity values were performed.

In the first-trimester, we found a statistically significant and positive correlation between placental elasticity values and bilateral uterine artery notching $(r=0.193, \mathrm{p}=0.045)$ and mean arterial pressure $(\mathrm{r}=0.398, \mathrm{p}<0.001)$. Likewise, positive correlation was found between placental elasticity values and bilateral uterine artery notching $(r=0.303, p=0.023)$ and mean arterial pressure $(\mathrm{r}=0.274, \mathrm{p}=0.041)$ in the second-trimester.

Our study showed that placental elasticity values were positively correlated with mean arterial pressure and bilateral uterine artery notching in the first and second-trimesters. In light of these findings, the utility of placental elasticity measurement in early gestation to predict pathologies due to insufficient placentation has to be proven with comprehensive studies.
\end{abstract}

Key Words: Elastography, Doppler, placenta, prenatal screening

\section{Introduction}

The maternal serum markers have been utilizing for prenatal screening for more than 40 years. First, the utility of alpha-fetoprotein (AFP) peptide has been demonstrated in the screening of neural tube defects (1). In the following years, human chorionic gonadotropin (HCG), unconjugated estriol (uE3), HCG $\beta$ subunit $(\beta$ hCG), pregnancy-associated plasma protein-A (PAPP-A) and inhibin A have been used for aneuploidy screen in combination with various ultrasonography markers (2). There are studies in the literature that these markers can be used in the prediction of pathologies due to insufficient placentation such as preeclampsia and intrauterine growth retardation (IUGR). The decreased PAPPA level in the first-trimester has a high predictive value in the prediction of early-onset preeclampsia
(3). Both, abnormally low or high free $\beta$-hCG levels were found to be associated with an increased risk of adverse pregnancy outcomes. (4). Similarly, there are studies that reported association between development of preeclampsia and IUGR and second-trimester screening markers $(5,6)$.

It has been known that there is an increase in the uterine artery resistance of pregnant women with preeclampsia and IUGR. Based on these findings, studies conducted in the first and second-trimester showed that the increase in uterine artery resistance was associated with early-onset preeclampsia and IUGR (7). Risk calculation programs for preeclampsia and IUGR have been developed with formulas using maternal factors, biochemical parameters and uterine artery pulsatility index (PI) $(3,8)$. 
Ultrasonographic elasticity measurement can be used to detect inflammation and fibrosis in the target tissue (9). Point shear wave elastography (pSWE) is a recent technology that generates shear waves in target tissue by focal tissue displacement using acoustic radiation force pulse (ARFI) technology and can calculate quantitative tissue elasticity. The utility of placental elastography has been evaluated in many pregnancy-related diseases (10-13). The role of first and second-trimester placental elasticity on the prediction of the diseases associated with insufficient placentation has not been studied yet. As a pioneer study, our aim was to evaluate the relationship between placental elasticity values and maternal serum markers and uterine artery Doppler parameters in the first and secondtrimester.

\section{Materials and Methods}

One hundred and eight cases were included in this study between January-June 2019. Multiple gestation, fetal chromosomal or structural abnormalities, threatened abortion, known placental pathology, chronic hypertension and diabetes mellitus were exclusion criteria for the study. In addition, due to the technical difficulty of measuring elasticity, cases with posterior wall placenta located downwards were excluded. The study was carried out in accordance with the Helsinki Declaration. The Human Ethics Committee of Manisa Celal Bayar University approved the study (date: 1 August 2018, number 20.478.486/35652). All participants gave a written informed consent.

After routine ultrasonography and combined test performed at 11-14 weeks of gestation, placental elastography was performed with C5-1 convex probe of Affiniti $70 \mathrm{G}$ (Philips Healthcare) ultrasound system using ElastPQ technique. Those who were followed-up, underwent the triple serum screening test one month later and placental elasticity was measured again. Elasticity measurements were performed on the same day with screening tests and, the sonographer was blinded to the serum marker results. The sonographer had 14 years of experience in grayscale and 1 year of experience in elastosonography. Demographic and clinical parameters were recorded during the first examination.

Elastography measurements were performed in a lying position. A region of interest box was positioned perpendicular to the probe and minimal pressure was applied to the transducer for the lowest variation. We obtained ten random measurements from 10 different areas of the placenta (Figure 1). Each ElastPQ measurement was indicated in kilopascal $(\mathrm{kPa})$ with standard deviation (SS). The measurement was rejected if the SS value was greater than $30 \%$ or the ElastPQ value was less than $1 \mathrm{kPa}$ to improve the reliability of the measurements. If 10 acceptable measurements could not be obtained in 15 trials with these criteria, the case was excluded. The mean elasticity value was calculated from the arithmetic mean of 10 values. The mechanical indices did not exceed 1.5 and thermal indices did not exceed 1.7. The duration of pSWE examination did not exceed $5 \mathrm{~min}$ in any case.

Serum levels of PAPP-A, free $\beta$-hCG, uE3, HCG and AFP markers were measured by chemiluminescent immunoassay method (Immulite 2000 Xpi, Siemens, Germany). The arithmetic means of left and right uterine artery PI and resistivity index (RI) were recorded as mean PI and RI values of left and right uterine arteries.

Statistical Analysis: The Statistical Package for the Social Sciences software version 20.0 was utilized for statistical analyses. The distributions of variables were assessed with the KolmogorovSmirnov test. The correlation analysis between elasticity values and categorical variables was performed by using point double series correlation coefficient. The relationship between the elasticity values and continuous variables was determined using Pearson's correlation coefficient. Statistical comparison between the two measurements in a subject was evaluated with dependent sample ttest. $\mathrm{P}<0.05$ was considered statistically significant.

\section{Results}

122 pregnant women were evaluated during the first-trimester combined test.14 women were excluded because of unsuccessful measurement and successful evaluation rate was $88.5 \%$. 71 pregnant women were included in the secondtrimester because 32 of 108 pregnant women did not come for follow up and 5 cases were evaluated as failed measurement. Success ratio was $100 \%$ for second-trimester assessment. Demographic characteristics, biochemistry and ultrasonography parameters of the subjects in the first-trimester are presented in Table 1. The mean placental elasticity was $3.66 \pm 1.66 \mathrm{kPa}$ in the first-trimester. First-trimester placental elasticity values were positively and statistically significantly 


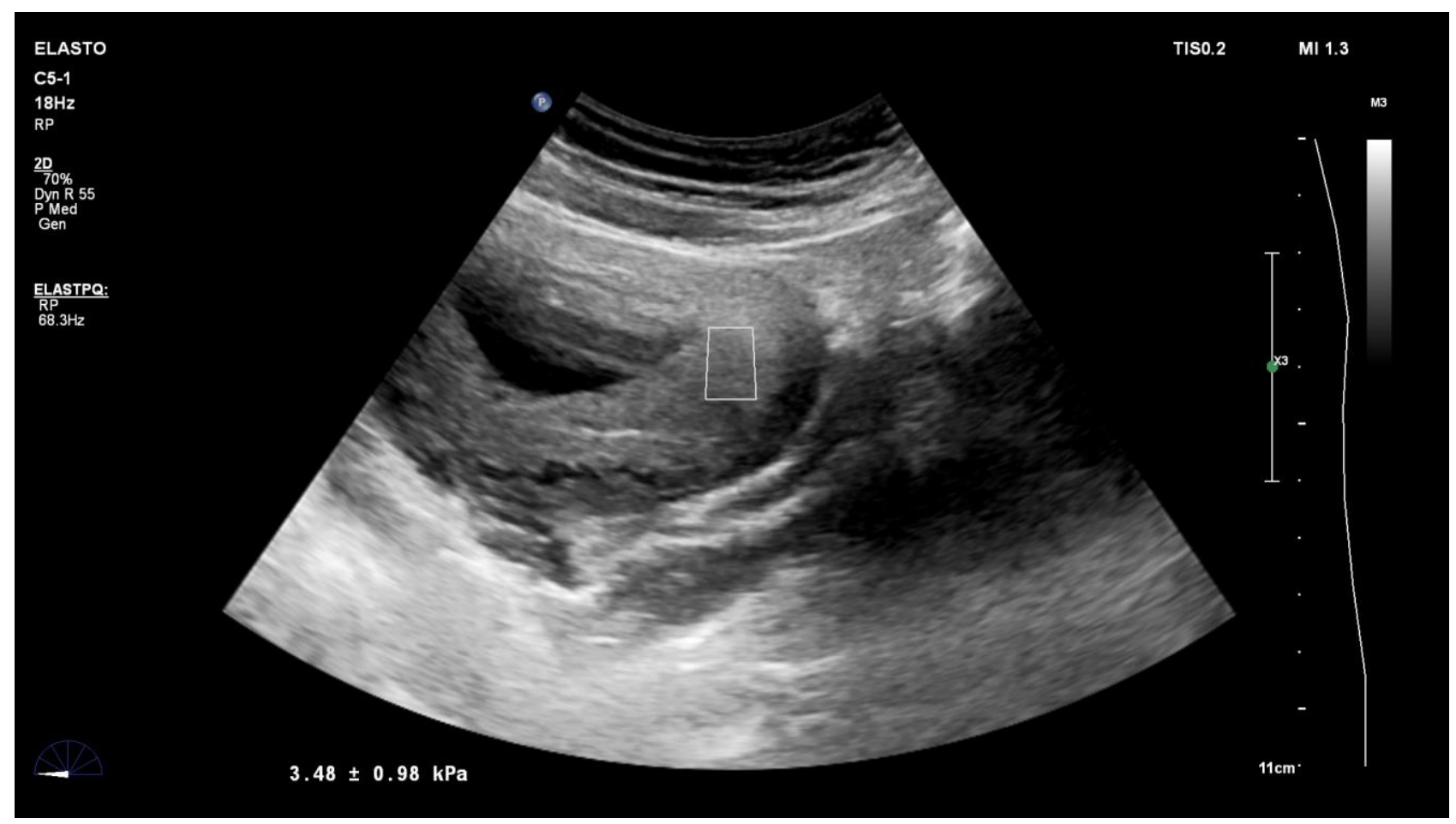

Fig. 1. Measurement of placenta elasticity using pSWE method

correlated with mean arterial pressure $(\mathrm{r}=0.398$, $\mathrm{p}<0.001)$ and notching in bilateral uterine arteries $(\mathrm{r}=0.193, \mathrm{p}=0.045) \quad($ Table 2$)$. There was no significant correlation between other parameters and placental elasticity.

Demographic characteristics, biochemistry and ultrasonography parameters for the secondtrimester evaluation are presented in Table 3. the mean placental elasticity was $3.37 \pm 1.22 \mathrm{kPa}$ in the second-trimester. The correlation analysis of second-trimester placental elasticity values and other parameters showed statistically significant and positive correlations between mean arterial pressure $(\mathrm{r}=0.274, \mathrm{p}=0.041)$ and notching in bilateral uterine arteries $(r=0.303, p=0.023$ ) (Table 4). There was no significant correlation between other parameters and placental elasticity.

The placental elasticity values of 71 subjects who participated in both assessments were evaluated by the dependent sample t-test. No significant difference was found between two assessments (3.76 \pm 1.69 vs $3.37 \pm 1.22, \mathrm{p}=0.122)$.

\section{Discussion}

In the current study, no correlation between was found between any maternal serum screening marker and placental elasticity values. Similarly, there was no correlation between mean uterine artery PI and RI values and placental elasticity. However, we found a significant correlation between placental elasticity and mean arterial pressure and a significant correlation between bilateral uterine artery notching and placental elasticity. Nevertheless, this study is the first to evaluate the relationship between placental elasticity and these parameters.

It was established that tissue stiffness could be altered in many pathological and physiological conditions. The evaluation of organs using ultrasonographic elastography as an additional method to conventional gray-scale ultrasonography is becoming widespread. In today's clinical practice, this method has been used in the diagnosis of liver fibrosis and breast lesions $(14,15)$. Different elastography methods have been developed over time. The ARFI method is the most recently introduced method, which is less dependent on the operator and gives more accurate results (16). The pSWE method, which we used in this study, provides fast and simple usage with ARFI technology.

There have been many publications in the literature about placental elasticity in recent years. The 2D shear wave elastography and pSWE enabled in-vivo elasticity measurement of the placenta. The safety aspects associated with ultrasonographic elastography are the same as for Doppler ultrasonography. It was reported that the mechanical effect and temperature rise generated by shear waves, were within the safe limits determined by the US Food and Drug Administration (17). No thermal or structural 
Table 1. Demographic variables, biochemical and ultrasonographic parameters of the cases in the firsttrimester

\begin{tabular}{lc}
\hline & $\begin{array}{c}\text { Subjects included in the study in the first-trimester } \\
(\mathrm{n}=108)\end{array}$ \\
\hline Age (year) & $29.55 \pm 5.44$ \\
Gravidity(n) & $2.4 \pm 1.64$ \\
Parity (n) & $0.89 \pm 1.08$ \\
BMI (kg / m2) & $24.24 \pm 3.57$ \\
CRL (mm) & $58.57 \pm 7.47$ \\
Mean arterial pressure (mmHg) & $92.29 \pm 2.6$ \\
Serum PAPP-A (mIU / ml) & $3.07 \pm 2.14$ \\
PAPP-A MoM & $1.06 \pm 0.53$ \\
Serum free BHCG (ng / ml) & $50.03 \pm 45.80$ \\
Free BHCG MoM & $1.22 \pm 1.02$ \\
Mean uterine artery PI & $1.83 \pm 0.5$ \\
Mean uterine artery RI & $0.75 \pm 0.09$ \\
Bilateral uterine artery notch $(\%)$ & $47.22(51 / 108)$ \\
Mean plasenta elasticity $(\mathrm{kPa})$ & $3.66 \pm 1.66$ \\
\hline
\end{tabular}

BMI body mass index, CRL crown-lump length, PAPP-A pregnancy associated plasma protein A, BHCG beta human chorionic gonadotropin, MoM multiples of median, PI pulsatility index, RI resistivity index, kPa kilopascal Data were presented as mean \pm standard deviation

Table 2. Correlation analysis of first-trimester placental elasticity values and other parameters

\begin{tabular}{lcc}
\hline & \multicolumn{2}{c}{ Placenta elasticity } \\
\hline Age & $\mathrm{r}$ & $\mathrm{p}$ \\
BMI & -0.042 & 0.664 \\
Mean arterial pressure & 0.155 & 0.108 \\
CRL & 0.398 & $<0.001$ \\
Serum PAPP-A & -0.004 & 0.968 \\
PAPP-A MoM & -0.114 & 0.246 \\
Serum free BHCG & -0.072 & 0.463 \\
Free BHCG MoM & -0.108 & 0.272 \\
Mean uterine artery PI & -0.1 & 0.307 \\
Mean uterine artery RI & 0.069 & 0.477 \\
Bilateral uterine artery notch & 0.073 & 0.453 \\
\hline
\end{tabular}

BMI body mass index, CRL crown-lump length, PAPP-A pregnancy associated plasma protein A, BHCG beta human chorionic gonadotropin, MoM multiples of median, PI pulsatility index, RI resistivity index

damage was reported in a study investigating the biological effect of elastography on the placenta (18). Nevertheless, larger studies are needed to conclude that the ARFI elastography is completely safe for the fetus. In the present study, ARFI waves were applied to the placenta and the fetuses were not within the pSWE wave pathway.

The prediction and prevention of diseases that are related to inadequate trophoblastic invasion and placentation are the most concentrated subjects of modern obstetrics. The efficacy of various maternal serum markers to predict pathologies related to inadequate placentation has been demonstrated $(19,20)$. Also, the mean uterine artery PI and bilateral uterine artery notch finding have high predictive value in pathologies related to impaired placentation (21). In the literature, studies on pregnant women with IUGR and preeclampsia have reported increased placental elasticity values in these patients $(10,11,22)$. In the light of this information, we hypothesized that the 
Table 3. Demographic variables, biochemical and ultrasonographic parameters of the cases in the second-trimester

\begin{tabular}{lc}
\hline & $\begin{array}{c}\text { Subjects included in the study in the second-trimester } \\
(\mathrm{n}=71)\end{array}$ \\
\hline EFW (gr) & $221.82 \pm 51.74$ \\
BMI (kg / m2) & $24.88 \pm 3.97$ \\
Mean arterial pressure (mmHg) & $91.37 \pm 1,81$ \\
Serum uE3 (ng / ml) & $0.72 \pm 0.27$ \\
uE3 MoM & $0.76 \pm 0.31$ \\
Serum AFP (IU / ml) & $46.5 \pm 62.83$ \\
AFP MoM & $1 \pm 0.42$ \\
Serum HCG (mIU / ml) & $23861.67 \pm 13039.8$ \\
HCG MoM & $1.08 \pm 0.63$ \\
Mean uterine artery PI & $1.5 \pm 0.38$ \\
Mean uterine artery RI & $0.7 \pm 0.08$ \\
Bilateral uterine artery notch $(\%)$ & $18.3(13 / 71)$ \\
Mean plasenta elasticity $(\mathrm{kPa})$ & $3.37 \pm 1.22$ \\
\hline
\end{tabular}

EFW estimated fetal weight, BMI body mass index, E3 estriol,AFP alfafetoprotein, HCG human chorionic gonadotropin, MoM multiples of median, PI pulsatility index, RI resistivity index, $\mathrm{kPa}$ kilopascal

Data were presented as mean \pm standard deviation

Table 4. Correlation analysis of second-trimester placental elasticity values and other parameters

\begin{tabular}{lcc}
\hline & \multicolumn{2}{c}{ Placenta elasticity } \\
\hline BMI & $\mathrm{R}$ & $\mathrm{p}$ \\
Mean arterial pressure & -0.052 & 0.634 \\
EFW & 0.274 & 0.041 \\
Serum uE3 & 0.14 & 0.263 \\
uE3 MoM & -0.172 & 0.166 \\
Serum AFP & -0.194 & 0.119 \\
AFP MoM & -0.055 & 0.664 \\
Serum HCG & -0.046 & 0.712 \\
HCG MoM & 0.036 & 0.775 \\
Mean uterine artery PI & 0.103 & 0.408 \\
Mean uterine artery RI & -0.124 & 0.365 \\
Bilateral uterine artery notch & -0.11 & 0.424 \\
\hline
\end{tabular}

EFW estimated fetal weight, BMI body mass index, E3 estriol,AFP alfafetoprotein, HCG human chorionic gonadotropin, MoM multiples of median, PI pulsatility index, RI resistivity index

measurement of placental elasticity may have a role in the prediction of these diseases in the early gestational weeks. For this purpose, before conducting a prospective study on the relationship between placental elasticity and poor obstetric outcomes in early pregnancy, we aimed to evaluate the possible relationship between the placental elasticity and serum markers that have been shown to be useful in predicting poor obstetric outcomes. We found significant positive correlations between the placental elasticity and bilateral uterine artery notching in both trimesters. We conclude that increased placental stiffness in early pregnancy may be associated with poor obstetric outcomes. However, our interpretation should be supported by prospective longitudinal studies. In pregnant women with preeclampsia, a positive correlation has been shown between placental elasticity and uterine artery PI and RI values (23). In our study, we did not find any significant relationship between these variables in early gestational weeks. 
In both trimesters, we found a positive and strong correlation between mean arterial pressure and placental elasticity. This result is noteworthy, considering the studies that reported the effectiveness of mean arterial pressure in the prediction of diseases associated with defective placentation $(24,25)$. In our study, we found that placental elasticity values did not change significantly between the two assessments. Longitudinal studies including both healthy pregnant women and women with defective placentation are needed to elucidate this finding.

The most important shortcoming of the study is that it does not include different groups due to its descriptive nature. Although demographic characteristics were homogeneous, the distinction between high and low risk pregnancies in terms of poor obstetric outcome could provide additional information. Nevertheless, this study provided significant results for future longitudinal studies as the first study evaluating the relationship between maternal demographic characteristics, serum markers, Doppler findings and placental elasticity in early gestational weeks.

In conclusion, we found that placental elasticity values in first and second-trimesters were positively correlated with bilateral uterine artery notching and mean arterial. In the light of these findings, we conclude that future studies that will investigate the utility of placental elasticity values in predicting the pathologies due to insufficient placentation may provide significant results.

Conflict of interest: Authors declare no conflict of interest.

\section{References}

1. Wald NJ, Cuckle H. Maternal serum alphafetoprotein measurement in antenatal screening for anencephaly and spina bifida in early pregnancy. Report of the U.K. Collaborative Study on Alpha-Fetoprotein in Relation to Neural-Tube Defects. Lancet 1977; 1323-1332.

2. Cuckle H, Maymon R. Development of prenatal screening--A historical overview. Semin Perinatol 2016; 40: 12-22.

3. Akolekar R, Syngelaki A, Poon L, Wright D, Nicolaides KH. Competing risks model in early screening for preeclampsia by biophysical and biochemical markers. Fetal Diagn Ther 2013; 33: 8-15.

4. Sirikunalai P, Wanapirak C, Sirichotiyakul S, et al. Associations between maternal serum free beta human chorionic gonadotropin ( $\beta$-hCG) levels and adverse pregnancy outcomes. J Obstet Gynaecol 2016; 36: 178-182.
5. Audibert F, Benchimol Y, Benattar C, Champagne C, Frydman R. Prediction of preeclampsia or intrauterine growth restriction by second trimester serum screening and uterine Doppler velocimetry. Fetal Diagn Ther 2005; 20 : 48-53.

6. Long W, Zhou Q, Wang H, et al. Secondtrimester Maternal Serum Screening Biomarkers in the Risk Assessment for Preeclampsia. Ann Clin Lab Sci 2018; 48: 308-313.

7. Velauthar L, Plana MN, Kalidindi M, et al. Firsttrimester uterine artery Doppler and adverse pregnancy outcome: a meta-analysis involving 55 974 women. Ultrasound Obstet Gynecol 2014; 43: 500-507.

8. Karagiannis G, Akolekar R, Sarquis R, Wright D, Nicolaides KH. Prediction of small-for-gestation neonates from biophysical and biochemical markers at 11-13 weeks. Fetal Diagn Ther 2011; 29: 148-154.

9. Ferraioli G, Maiocchi L, Lissandrin R, Tinelli C, De Silvestri A, Filice C. Accuracy of the ElastPQ technique for the assessment of liver fibrosis in patients with chronic hepatitis C: a "Real life" single center study. J Gastrointestin Liver Dis 2016; 25: 331-335.

10. Karaman E, Arslan H, Çetin O, et al. Comparison of placental elasticity in normal and pre-eclamptic pregnant women by acoustic radiation force impulse elastosonography. J Obstet Gynaecol Res 2016; 42: 1464-1470.

11. Akbas M, Koyuncu FM, Artunç-Ulkumen B. Placental elasticity assessment by point shear wave elastography in pregnancies with intrauterine growth restriction. J Perinat Med 2019; 47: 841846.

12. Yuksel MA, Kilic F, Kayadibi Y, et al. Shear wave elastography of the placenta in patients with gestational diabetes mellitus. J Obstet Gynaecol 2016; 36: 585-588.

13. Cetin O, Karaman E, Arslan H, Akbudak I, Yildizhan R, Kolusar1 A. Acoustic radiation force impulse elastosonography of placenta in maternal red blood cell alloimmunization: a preliminary and descriptive study. Med Ultrason 2017;19: 73-78.

14. Barr RG, Nakashima K, Amy D, et al. WFUMB guidelines and recommendations for clinical use of ultrasound elastography. Ultrasound Med Biol 2015; 41: 1148-1160.

15. Ferraioli G, Filice C, Castera L, et al. WFUMB guidelines and recommendations for clinical use of ultrasound elastography. Ultrasound Med Biol 2015; 41: 1161-1179.

16. Sigrist R, Liau J, Kaffas AE, Chammas MC, Willmann JK. Ultrasound elastography: review of techniques and clinical applications. Theranostics 2017; 7: 1303-1329.

17. Herman BA, Harris GR. Models and regulatory considerations for transient temperature rise during diagnostic ultrasound pulses. Ultrasound Med Biol 2002; 28: 1217-1224. 
18. Sugitani M, Fujita Y, Yumoto $Y$, et al. A new method for measurement of placental elasticity: acoustic radiation force impulse imaging. Placenta 2013; 34: 1009-1013.

19. Poon LC, Nicolaides KH. First-trimester maternal factors and biomarker screening for preeclampsia. Prenat Diagn 2014; 34: 618-627.

20. Levine RJ, Maynard SE, Qian C, et al. Circulating angiogenic factors and the risk of preeclampsia. N Engl J Med 2004; 350: 672683.

21. Cnossen JS, Morris RK, ter Riet G, et al. Use of uterine artery Doppler ultrasonography to predict pre-eclampsia and intrauterine growth restriction: a systematic review and bivariable meta-analysis. CMAJ 2008; 178: 701-711.
22. Arioz Habibi H, Alici Davutoglu E, Kandemirli SG, et al. In vivo assessment of placental elasticity in intrauterine growth restriction by shear-wave elastography. Eur J Radiol 2017; 97: 16-20.

23. Kılıç F, Kayadibi Y, Yüksel MA, et al. Shear wave elastography of placenta: in vivo quantitation of placental elasticity in preeclam psia. Diagn Interv Radiol 2015; 21: 202-207.

24. Akolekar R, Syngelaki A, Sarquis R, Zvanca M, Nicolaides KH. Prediction of early, intermediate and late pre-eclampsia from maternal factors, biophysical and biochemical markers at 11-13 weeks. Prenat Diagn 2011; 31: 66-74.

25. Kuc S, Koster MP, Franx A, Schielen PC, Visser GH. Maternal characteristics, mean arterial pressure and serum markers in early prediction of preeclampsia. PLoS One 2013; 8: 63546. 